Supplement of Atmos. Chem. Phys., 21, 16631-16644, 2021

https://doi.org/10.5194/acp-21-16631-2021-supplement

(c) Author(s) 2021. CC BY 4.0 License.

(c) (1)

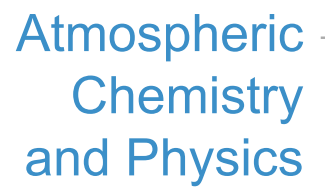

Supplement of

\title{
Measurement report: Molecular characteristics of cloud water in southern China and insights into aqueous-phase processes from Fourier transform ion cyclotron resonance mass spectrometry
}

Wei Sun et al.

Correspondence to: Xinhui Bi (bixh@gig.ac.cn)

The copyright of individual parts of the supplement might differ from the article licence. 
During the investigated period (May 11-12), air masses from the South China Sea and Southeast Asia and across Hainan Island and the boundary between Guangdong and Guangxi Provinces were dominant, according to the back-trajectory analysis (Fig. S1). As presented in Fig. S2, there is no obvious diurnal variation of temperature $\left(15.2-15.9{ }^{\circ} \mathrm{C}\right)$. As expected, the relative humidity was stable, with a constant value of $100 \%$. The wind with speeds ranged from 3.3 to $7.5 \mathrm{~m} \mathrm{~s}^{-1}$ and

30 primarily came from the southwest. The $\mathrm{PM}_{2.5}$ concentration varied from 2.0 to $4.3 \mu \mathrm{g} \mathrm{m}^{-3}$, much lower than that in most urban areas in southern China. The $\mathrm{NO}_{\mathrm{x}}, \mathrm{O}_{3}$, and $\mathrm{SO}_{2}$ concentrations ranged from 2.2 to $7.7 \mu \mathrm{g} \mathrm{m}^{-3}, 60.6$ to $101.0 \mu \mathrm{g} \mathrm{m}^{-3}$, and 0.2 to $0.8 \mu \mathrm{g} \mathrm{m}^{-3}$, respectively. The $\mathrm{NO}_{\mathrm{x}}$ and $\mathrm{O}_{3}$ concentrations showed a clear diurnal variation. Both of them were relatively stable in the daytime (approximately 2-4 $\mu \mathrm{g} \mathrm{m}^{-3}$ and 85-100 $\mu \mathrm{g} \mathrm{m}^{-3}$ for $\mathrm{NO}_{\mathrm{x}}$ and $\mathrm{O}_{3}$, respectively). However, $\mathrm{NO}_{\mathrm{x}}$ reached a peak of $8 \mu \mathrm{g} \mathrm{m}^{-3}$ at approximately 01:00 LT, May 12, and then decreased, whereas $\mathrm{O}_{3}$ dropped from approximately

$35100 \mu \mathrm{g} \mathrm{m}^{-3}$ at about 21:00, May 11 to $60 \mu \mathrm{g} \mathrm{m}^{-3}$ in the next morning (Fig. S2).

The concentrations of inorganic ions in cloud water are listed in Table $\mathrm{S} 1 . \mathrm{Na}^{+}, \mathrm{NH}_{4}^{+}, \mathrm{K}^{+}, \mathrm{Ca}^{2+}, \mathrm{Mg}^{2+}, \mathrm{Cl}^{-}, \mathrm{NO}_{3}{ }^{-}$, and $\mathrm{SO}_{4}{ }^{2-}$ were detected. $\mathrm{SO}_{4}{ }^{2-}, \mathrm{NO}_{3}{ }^{-}$, and $\mathrm{NH}_{4}^{+}$were dominant, with average concentrations of $3.53,2.88$, and $1.11 \mathrm{mg} \mathrm{L}^{-1}$, respectively; together, they contributed approximately $90 \%$ of the total water-soluble inorganic ions. These concentrations are comparable to those observed in cloud water collected at Mt. Heng (Sun et al., 2010) and Mt. Lu (Sun et al., 2016) in southern China, but much lower than those observed at Mt. Tai in northern China (Guo et al., 2012).

\section{References}

Guo, J., Wang, Y., Shen, X., Wang, Z., Lee, T., Wang, X., Li, P., Sun, M., Collett, J. L., Wang, W., and Wang, T.: Characterization of cloud water chemistry at Mount Tai, China: Seasonal variation, anthropogenic impact, and cloud processing, Atmos. Environ., 60, 467-476, https://doi.org/10.1016/j.atmosenv.2012.07.016, 2012.

Sun, M., Wang, Y., Wang, T., Fan, S., Wang, W., Li, P., Guo, J., and Li, Y.: Cloud and the corresponding precipitation chemistry in south China: Water-soluble components and pollution transport, J. Geophys. Res., 115, https://doi.org/10.1029/2010jd014315, 2010.

Sun, X., Wang, Y., Li, H., Yang, X., Sun, L., Wang, X., Wang, T., and Wang, W.: Organic acids in cloud water and rainwater at a mountain site in acid rain areas of South China, Environ. Sci. Pollut. Res. Int., 23, 9529-9539, https://doi.org/10.1007/s11356-016-6038-1, 2016. 


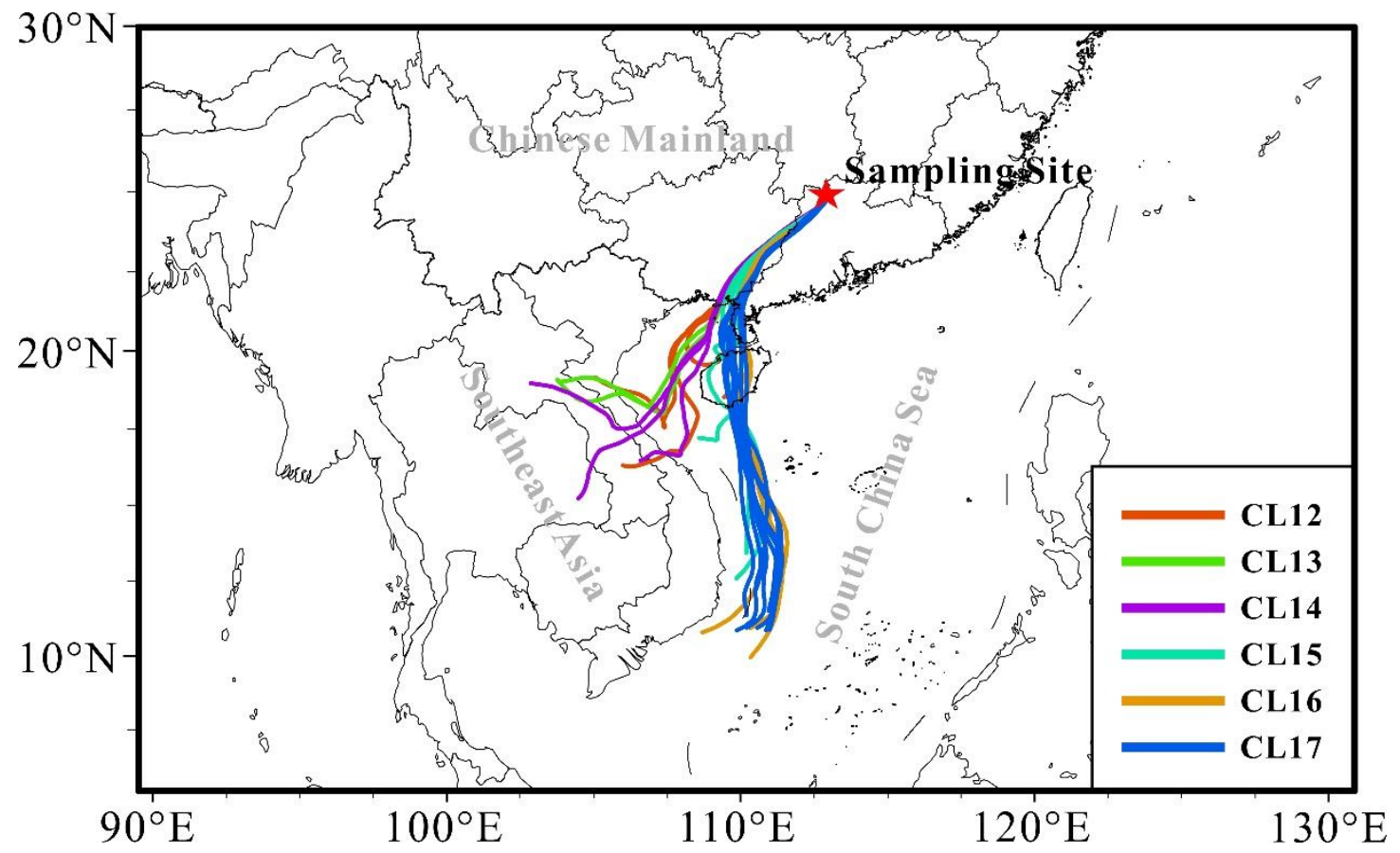

Figure S1. The location of the sampling site and $72 \mathrm{~h}$ back trajectories displayed by the Hybrid Single-Particle Lagrangian Integrated Trajectory model for every hours during sampling time (May 11 10:00 - May 12 08:00 LT). 


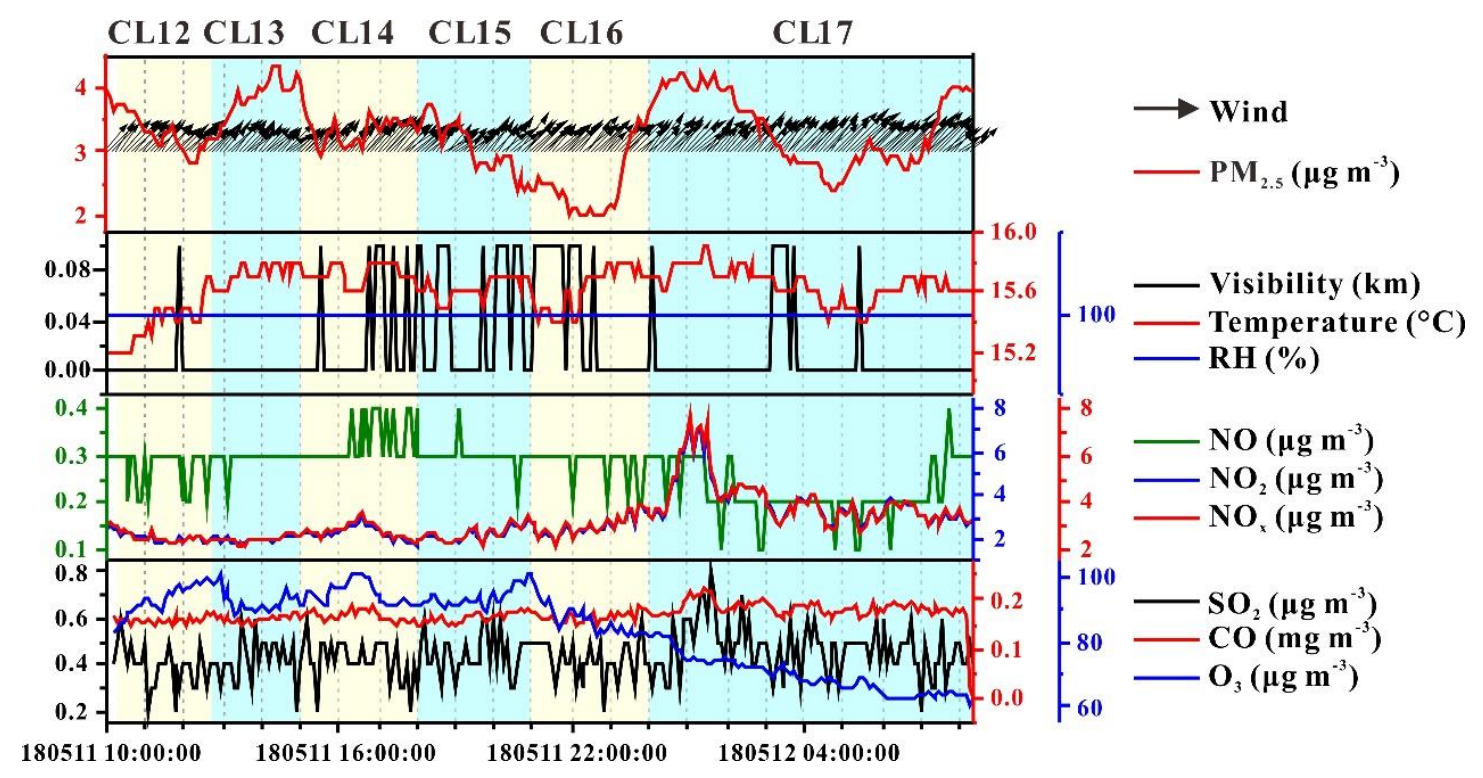

Figure S2. The time series of the meteorological data including visibility, temperature, relative humidity, concentration of some 60 contaminant in the atmosphere $\left(\mathrm{NO}, \mathrm{NO}_{2}, \mathrm{NO}_{\mathbf{x}}, \mathrm{SO}_{2}, \mathbf{C O}\right.$, and $\mathrm{O}_{3}$ ). The data was obtained from Guangdong Environmental Monitoring Center. The alternating color lumps in the figure represent the sampling interval. 


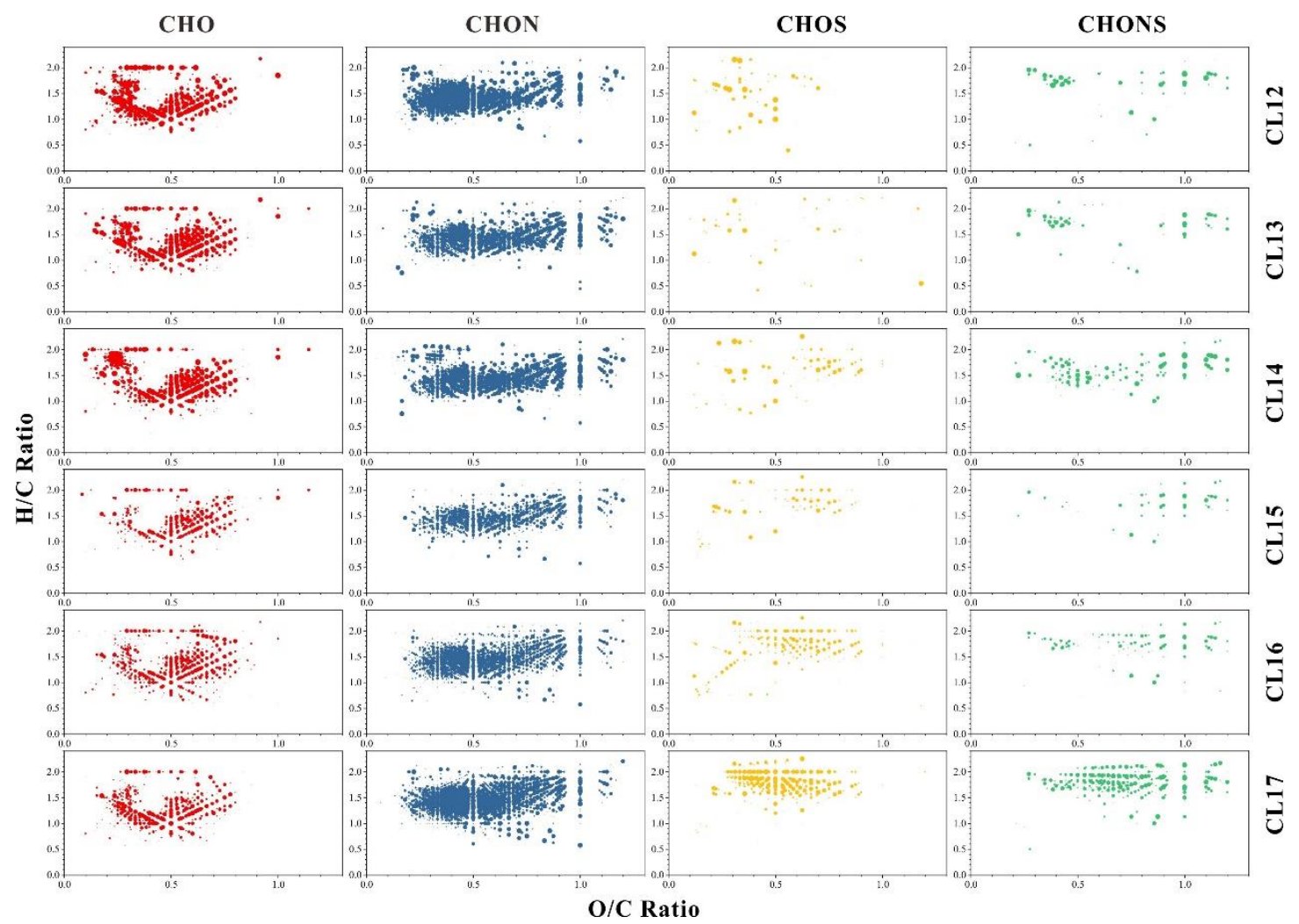

Figure S3. Van Krevelen diagrams as a function of four groups (CHO, CHNO, CHOS, and CHNOS) for the cloud water samples.

The larger point in the diagram represents the higher relative abundance of the formula. 

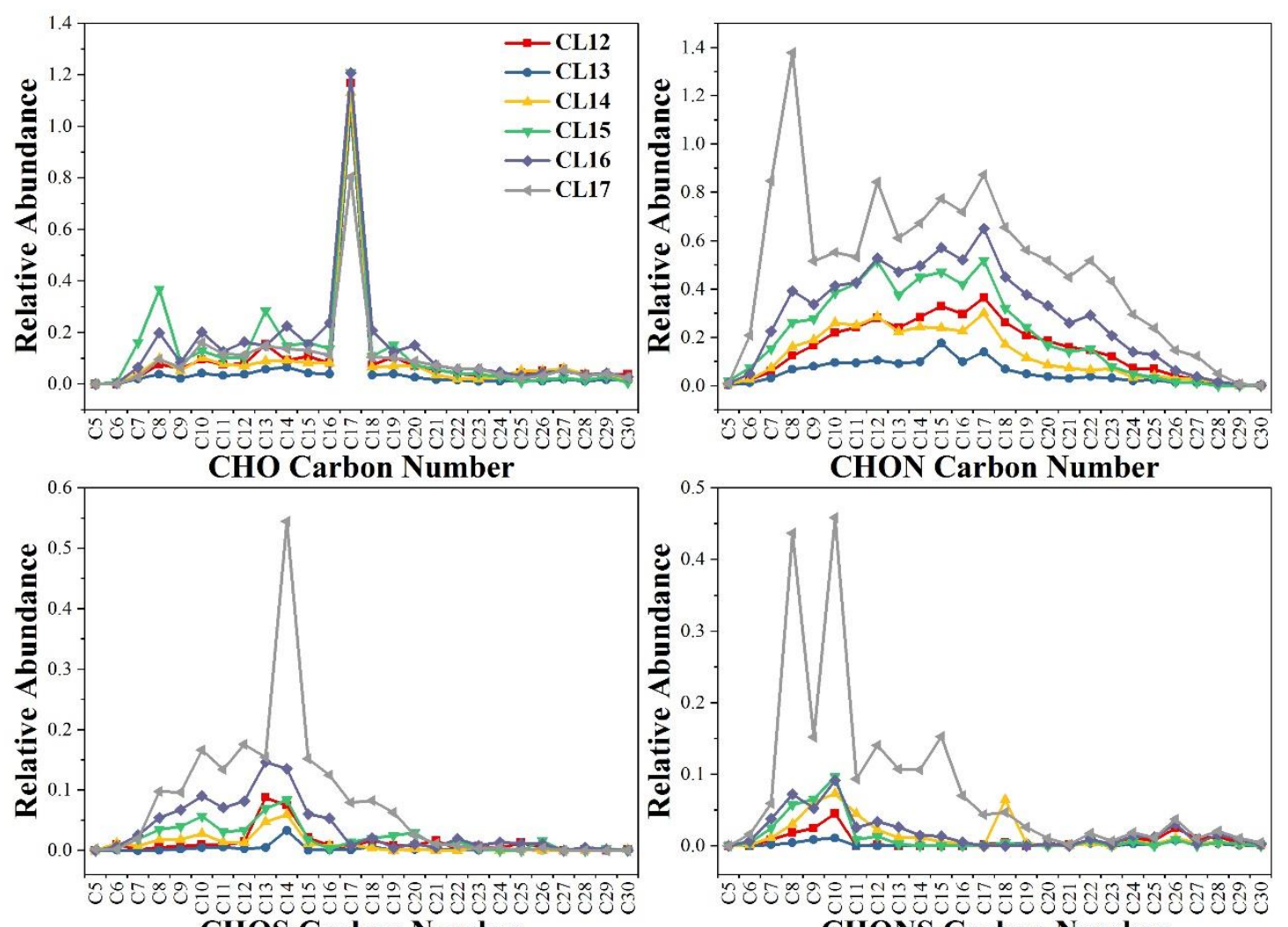

Figure S4. The relative abundances with respect to the carbon atom number within four groups (CHO, CHON, CHOS, and CHONS) of the molecular formulas in the cloud water samples. 

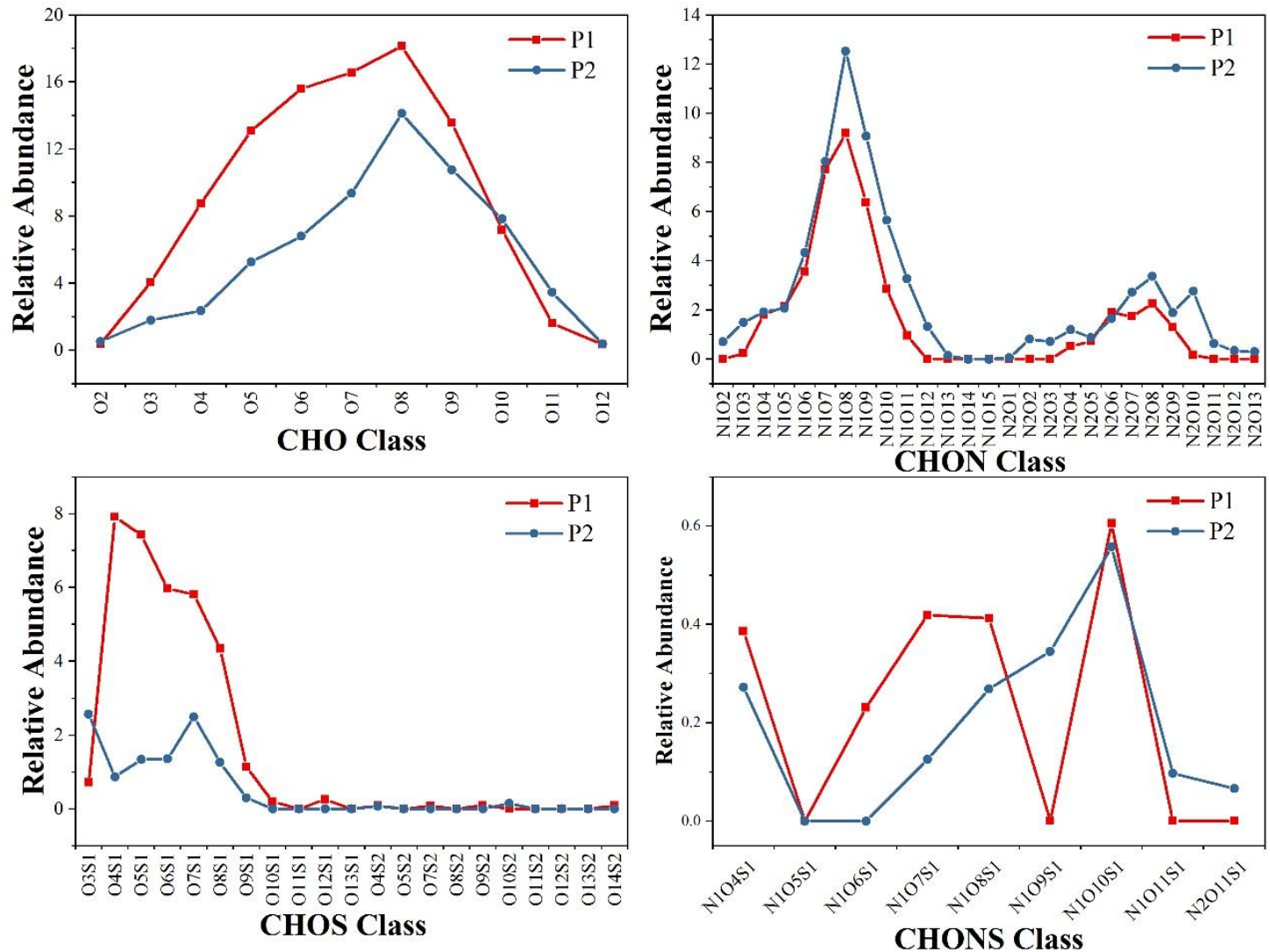

Figure S5. Relative abundance of the categories of CHO, CHON, CHOS and CHONS formulas according to the characteristic atom groups in the molecular formulas in two PM2.5 Samples (P1 and P2). 
75 Table S1. The concentration of water-soluble inorganic ions in cloud water. The unit of the data is $\mathrm{mg} \mathrm{L}^{-1}$.

\begin{tabular}{cccccccccc}
\hline Sample ID & $\mathrm{Na}^{+}$ & $\mathrm{NH}_{4}^{+}$ & $\mathrm{K}^{+}$ & $\mathrm{Mg}^{2+}$ & $\mathrm{Ca}^{2+}$ & $\mathrm{Cl}^{-}$ & $\mathrm{NO}_{3}^{-}$ & $\mathrm{SO}_{4}^{2-}$ & $\mathrm{C}_{2} \mathrm{O}_{4}^{2-}$ \\
\hline CL12 & 0.17 & 0.61 & 0.04 & 0.04 & 0.01 & 0.34 & 2.21 & 2.66 & 0.19 \\
CL13 & 0.27 & 0.80 & 0.09 & 0.03 & 0.02 & 0.27 & 2.30 & 2.74 & 0.30 \\
CL14 & 0.23 & 0.91 & 0.05 & 0.03 & N.D. & 0.27 & 2.30 & 2.74 & 0.47 \\
CL15 & 0.29 & 1.23 & 0.08 & 0.05 & 0.04 & 0.37 & 3.11 & 4.05 & 0.39 \\
CL16 & 0.25 & 1.46 & 0.09 & 0.05 & 0.03 & 0.46 & 3.44 & 4.23 & 0.06 \\
CL17 & 0.37 & 1.69 & 0.10 & 0.06 & 0.03 & 0.67 & 3.93 & 4.74 & N.D. \\
\hline
\end{tabular}

N.D.: Not detected. 
Table S2. The number fraction and relative abundance fraction of four groups (CHO, CHON, CHOS, and CHONS) in each cloud water sample.

\begin{tabular}{c|cccc|cccc}
\hline \multirow{2}{*}{$\begin{array}{c}\text { Sample } \\
\text { ID }\end{array}$} & \multicolumn{4}{|c|}{ Number Fraction } & \multicolumn{3}{c}{ Relative Abundance Fraction } \\
\cline { 2 - 8 } & CHO & CHON & CHOS & CHONS & CHO & CHON & CHOS & CHONS \\
\hline CL12 & $26.6 \%$ & $65.2 \%$ & $4.5 \%$ & $3.7 \%$ & $37.6 \%$ & $54.9 \%$ & $4.7 \%$ & $2.7 \%$ \\
CL13 & $28.3 \%$ & $64.4 \%$ & $3.6 \%$ & $3.7 \%$ & $52.1 \%$ & $43.6 \%$ & $2.3 \%$ & $2.0 \%$ \\
CL14 & $27.1 \%$ & $60.2 \%$ & $4.9 \%$ & $7.8 \%$ & $39.3 \%$ & $50.5 \%$ & $4.1 \%$ & $6.1 \%$ \\
CL15 & $23.5 \%$ & $66.2 \%$ & $6.4 \%$ & $3.9 \%$ & $35.3 \%$ & $56.2 \%$ & $5.3 \%$ & $3.1 \%$ \\
CL16 & $21.7 \%$ & $65.4 \%$ & $8.1 \%$ & $4.8 \%$ & $30.0 \%$ & $58.9 \%$ & $7.3 \%$ & $3.9 \%$ \\
CL17 & $16.3 \%$ & $65.1 \%$ & $9.4 \%$ & $9.3 \%$ & $13.8 \%$ & $65.3 \%$ & $10.2 \%$ & $10.8 \%$ \\
\hline
\end{tabular}

80 
Table S3. The number fraction and relative abundance fraction of four groups (CHO, CHON, CHOS, and CHONS) in two PM 2.5 samples.

\begin{tabular}{c|cccc|cccc}
\hline \multirow{2}{*}{ Sample ID } & \multicolumn{4}{|c|}{ Number Fraction } & \multicolumn{4}{c}{ Relative Abundance Fraction } \\
\cline { 2 - 8 } & CHO & CHON & CHOS & CHONS & CHO & CHON & CHOS & CHONS \\
\hline P1 & $49.8 \%$ & $31.8 \%$ & $17.2 \%$ & $1.2 \%$ & $55.5 \%$ & $24.3 \%$ & $19.1 \%$ & $1.2 \%$ \\
P2 & $39.9 \%$ & $51.0 \%$ & $7.5 \%$ & $1.6 \%$ & $44.0 \%$ & $47.3 \%$ & $7.5 \%$ & $1.2 \%$ \\
\hline
\end{tabular}




\begin{tabular}{|c|c|c|c|c|c|c|c|c|c|c|}
\hline \multicolumn{11}{|c|}{ All } \\
\hline Sample ID & $\mathrm{O} / \mathrm{C}$ & $\mathrm{H} / \mathrm{C}$ & DBE & \#C & $\mathrm{OS}_{\mathrm{C}}$ & $\mathrm{O} / \mathrm{Cw}$ & $\mathrm{H} / \mathrm{Cw}$ & DBEw & \#CW & $\mathrm{OS}_{\mathrm{Cw}}$ \\
\hline CL12 & 0.46 & 1.44 & 6.16 & 16.92 & -0.88 & 0.45 & 1.47 & 5.70 & 16.16 & -0.87 \\
\hline CL13 & 0.52 & 1.45 & 5.96 & 16.31 & -0.76 & 0.45 & 1.48 & 5.37 & 15.82 & -0.81 \\
\hline CL14 & 0.53 & 1.46 & 5.61 & 15.91 & -0.82 & 0.51 & 1.48 & 5.25 & 15.22 & -0.79 \\
\hline CL15 & 0.56 & 1.47 & 5.61 & 15.20 & -0.76 & 0.56 & 1.49 & 5.10 & 14.38 & -0.72 \\
\hline CL16 & 0.52 & 1.46 & 5.92 & 16.47 & -0.83 & 0.54 & 1.48 & 5.41 & 15.28 & -0.79 \\
\hline CL17 & 0.51 & 1.49 & 5.86 & 16.80 & -0.92 & 0.55 & 1.44 & 5.44 & 14.58 & -0.91 \\
\hline \multicolumn{11}{|c|}{ CHO } \\
\hline Sample ID & $\mathrm{O} / \mathrm{C}$ & $\mathrm{H} / \mathrm{C}$ & DBE & \#C & $\mathrm{OS}_{\mathrm{C}}$ & $\mathrm{O} / \mathrm{Cw}$ & $\mathrm{H} / \mathrm{Cw}$ & DBEw & $\# \mathrm{Cw}$ & $\mathrm{OS}_{\mathrm{Cw}}$ \\
\hline CL12 & 0.41 & 1.38 & 6.37 & 18.18 & -0.57 & 0.36 & 1.45 & 5.59 & 16.98 & -0.72 \\
\hline CL13 & 0.46 & 1.38 & 6.30 & 17.67 & -0.46 & 0.34 & 1.47 & 5.33 & 16.69 & -0.80 \\
\hline CL14 & 0.44 & 1.41 & 5.82 & 17.98 & -0.53 & 0.38 & 1.46 & 5.29 & 16.78 & -0.71 \\
\hline CL15 & 0.50 & 1.39 & 5.79 & 15.93 & -0.39 & 0.46 & 1.47 & 4.96 & 14.94 & -0.54 \\
\hline CL16 & 0.47 & 1.36 & 6.35 & 17.53 & -0.41 & 0.45 & 1.46 & 5.27 & 16.11 & -0.56 \\
\hline CL17 & 0.43 & 1.31 & 6.85 & 17.50 & -0.46 & 0.39 & 1.36 & 6.12 & 16.44 & -0.58 \\
\hline \multicolumn{11}{|c|}{ CHON } \\
\hline Sample ID & $\mathrm{O} / \mathrm{C}$ & $\mathrm{H} / \mathrm{C}$ & DBE & $\# \mathrm{C}$ & $\mathrm{OS}_{\mathrm{C}}$ & $\mathrm{O} / \mathrm{Cw}$ & $\mathrm{H} / \mathrm{Cw}$ & DBEw & \#Cw & $\mathrm{OS}_{\mathrm{Cw}}$ \\
\hline CL12 & 0.48 & 1.45 & 6.24 & 16.46 & -0.98 & 0.51 & 1.45 & 6.00 & 15.65 & -0.92 \\
\hline CL13 & 0.54 & 1.45 & 5.99 & 15.66 & -0.86 & 0.59 & 1.48 & 5.50 & 14.75 & -0.78 \\
\hline CL14 & 0.55 & 1.46 & 5.84 & 15.64 & -0.87 & 0.60 & 1.46 & 5.50 & 14.46 & -0.76 \\
\hline CL15 & 0.57 & 1.47 & 5.81 & 15.25 & -0.84 & 0.62 & 1.48 & 5.44 & 14.27 & -0.76 \\
\hline CL16 & 0.53 & 1.46 & 6.24 & 16.60 & -0.90 & 0.57 & 1.44 & 5.88 & 15.23 & -0.83 \\
\hline CL17 & 0.50 & 1.46 & 6.41 & 17.31 & -0.94 & 0.56 & 1.37 & 6.09 & 14.77 & -0.87 \\
\hline \multicolumn{11}{|c|}{ CHOS } \\
\hline Sample ID & $\mathrm{O} / \mathrm{C}$ & $\mathrm{H} / \mathrm{C}$ & DBE & $\# \mathrm{C}$ & $\mathrm{OS}_{\mathrm{C}}$ & $\mathrm{O} / \mathrm{Cw}$ & $\mathrm{H} / \mathrm{Cw}$ & DBEw & \#Cw & $\mathrm{OS}_{\mathrm{Cw}}$ \\
\hline CL12 & 0.39 & 1.56 & 4.92 & 16.20 & -1.22 & 0.36 & 1.66 & 3.87 & 15.11 & -1.39 \\
\hline
\end{tabular}




\begin{tabular}{|c|c|c|c|c|c|c|c|c|c|c|}
\hline CL13 & 0.50 & 1.58 & 4.75 & 16.16 & -1.03 & 0.45 & 1.56 & 4.58 & 15.15 & -1.11 \\
\hline CL14 & 0.59 & 1.58 & 3.81 & 12.35 & -0.99 & 0.50 & 1.66 & 3.19 & 12.60 & -1.21 \\
\hline CL15 & 0.50 & 1.61 & 4.35 & 13.93 & -1.11 & 0.47 & 1.68 & 3.72 & 13.77 & -1.23 \\
\hline CL16 & 0.52 & 1.65 & 3.64 & 13.74 & -1.09 & 0.51 & 1.71 & 3.21 & 13.27 & -1.18 \\
\hline CL17 & 0.50 & 1.74 & 2.89 & 14.10 & -1.21 & 0.48 & 1.75 & 2.72 & 13.43 & -1.27 \\
\hline \multicolumn{11}{|c|}{ CHONS } \\
\hline Sample ID & $\mathrm{O} / \mathrm{C}$ & $\mathrm{H} / \mathrm{C}$ & DBE & $\# \mathrm{C}$ & $\mathrm{OS}_{\mathrm{C}}$ & $\mathrm{O} / \mathrm{Cw}$ & $\mathrm{H} / \mathrm{Cw}$ & DBEw & $\# \mathrm{Cw}$ & $\mathrm{OS}_{\mathrm{Cw}}$ \\
\hline CL12 & 0.68 & 1.63 & 4.63 & 17.03 & -1.14 & 0.69 & 1.67 & 4.29 & 16.96 & -1.19 \\
\hline CL13 & 0.67 & 1.71 & 4.12 & 17.42 & -1.21 & 0.67 & 1.71 & 4.13 & 17.28 & -1.21 \\
\hline CL14 & 0.67 & 1.58 & 4.22 & 13.10 & -1.31 & 0.65 & 1.60 & 4.25 & 13.22 & -1.33 \\
\hline CL15 & 0.84 & 1.72 & 3.18 & 11.98 & -1.15 & 0.88 & 1.72 & 3.01 & 10.92 & -1.14 \\
\hline CL16 & 0.74 & 1.71 & 3.48 & 14.47 & -1.17 & 0.78 & 1.68 & 3.42 & 13.33 & -1.16 \\
\hline CL17 & 0.70 & 1.77 & 3.29 & 14.75 & -1.27 & 0.78 & 1.70 & 3.19 & 12.09 & -1.21 \\
\hline
\end{tabular}


Table S5. The relative-abundance-weighted average values of $\mathrm{O} / \mathrm{C}, \mathrm{H} / \mathrm{C}, \mathrm{DBE}, \mathrm{OS}_{\mathrm{C}}$ and for all formulas, $\mathrm{CHO}, \mathrm{CHON}, \mathrm{CHOS}$ and CHONS formulas in two PM2.5 samples (P1 and P2).

\begin{tabular}{cc|cccc}
\hline \multirow{2}{*}{ Group } & $\begin{array}{c}\text { Sample } \\
\text { ID }\end{array}$ & ${\mathrm{O} / \mathrm{C}_{\mathrm{w}}}$ & $\mathrm{H} \mathrm{C}_{\mathrm{w}}$ & $\mathrm{DBE}_{\mathrm{w}}$ & $\mathrm{OS}_{\mathrm{Cw}}$ \\
\hline \multirow{2}{*}{ All } & $\mathrm{P} 1$ & 0.45 & 1.53 & 5.04 & -0.84 \\
& $\mathrm{P} 2$ & 0.56 & 1.40 & 4.74 & -0.61 \\
\hline \multirow{2}{*}{ CHO } & $\mathrm{P} 1$ & 0.40 & 1.49 & 5.48 & -0.69 \\
& $\mathrm{P} 2$ & 0.53 & 1.45 & 5.03 & -0.40 \\
\hline \multirow{2}{*}{ CHON } & $\mathrm{P} 1$ & 0.53 & 1.51 & 5.32 & -0.89 \\
& $\mathrm{P} 2$ & 0.63 & 1.49 & 5.04 & -0.75 \\
\hline \multirow{2}{*}{ CHOS } & $\mathrm{P} 1$ & 0.46 & 1.65 & 3.49 & -1.19 \\
& $\mathrm{P} 2$ & 0.61 & 1.39 & 4.73 & -0.83 \\
\hline \multirow{2}{*}{ CHONS } & $\mathrm{P} 1$ & 0.66 & 1.69 & 3.22 & -1.40 \\
& $\mathrm{P} 2$ & 0.86 & 1.74 & 2.98 & -1.17 \\
\hline
\end{tabular}


Table S6. The fraction in relative abundance of aliphatic/olefinic and (condensed) aromatic structures in six cloud water samples.

\begin{tabular}{c|cccccc}
\hline \multicolumn{7}{c}{ CHO } \\
\hline Sample ID & CL12 & CL13 & CL14 & CL15 & CL16 & CL17 \\
\hline Aliphatic/Olefinic & $95.8 \%$ & $98.3 \%$ & $97.1 \%$ & $97.2 \%$ & $94.9 \%$ & $91.1 \%$ \\
(Condensed) Aromatic & $4.2 \%$ & $1.7 \%$ & $2.9 \%$ & $2.8 \%$ & $5.1 \%$ & $8.9 \%$ \\
\hline \multicolumn{7}{c}{ CHON } \\
\hline Sample ID & CL12 & CL13 & CL14 & CL15 & CL16 & CL17 \\
\hline Aliphatic/Olefinic & $96.1 \%$ & $97.5 \%$ & $97.2 \%$ & $97.1 \%$ & $92.5 \%$ & $79.2 \%$ \\
(Condensed) Aromatic & $3.9 \%$ & $2.5 \%$ & $2.8 \%$ & $2.9 \%$ & $7.5 \%$ & $20.8 \%$ \\
\hline \multicolumn{7}{c}{ CHOS } \\
\hline Sample ID & CL12 & CL13 & CL14 & CL15 & CL16 & CL17 \\
\hline Aliphatic/Olefinic & $93.9 \%$ & $95.3 \%$ & $96.3 \%$ & $93.5 \%$ & $95.7 \%$ & $98.8 \%$ \\
(Condensed) Aromatic & $6.1 \%$ & $4.7 \%$ & $3.7 \%$ & $6.5 \%$ & $4.3 \%$ & $1.2 \%$ \\
\hline \multicolumn{7}{c}{ CHONS } \\
\hline Sample ID & CL12 & CL13 & CL14 & CL15 & CL16 & CL17 \\
\hline Aliphatic/Olefinic & $95.7 \%$ & $98.2 \%$ & $100.0 \%$ & $100.0 \%$ & $98.6 \%$ & $99.6 \%$ \\
(Condensed) Aromatic & $4.3 \%$ & $1.8 \%$ & $0.0 \%$ & $0.0 \%$ & $1.4 \%$ & $0.4 \%$ \\
\hline
\end{tabular}

